\title{
INFLUÊNCIA DO ASPECTO NUTRICIONAL DE Eucalyptus grandis W. Hill ex Maiden NA PREFERÊNCIA ALIMENTAR DA LAGARTA DESFOLHADORA Eupseudosoma involuta (LEPIDOPTERA - ARCTIIDAE) ${ }^{1}$
}

\author{
Flávia Maria da Silva Carmo e Pedro Henrique Silva Penedo ${ }^{2}$
}

\begin{abstract}
RESUMO - O objetivo deste trabalho foi investigar se o consumo de folhas de mudas de eucalipto por insetos herbívoros pode ser influenciado pela condição nutricional a que essas plantas estão submetidas. Para tal, mudas de Eucalyptus grandis W. Hill ex Maiden foram mantidas em soluções nutritivas, constituindo tratamentos diferenciados entre si pela presença e ausência dos macronutrrientes nitrogênio, fósforo e potássio, além da solução completa e da testemunha, sendo este último tratamento constituído somente por água destilada. Após as plantas estarem submetidas às diferentes condições de nutrição por 30 dias, suas folhas foram arrancadas e oferecidas como dieta a lagartas de Eupseudossoma involuta (Lepidoptera - Arctiidae). Decorridas 20 horas do início do período de alimentação, o restante da dieta de folhas foi pesado. Avaliaram-se os parâmetros altura e número de folhas das mudas de E. grandis antes e depois de submetidas aos tratamentos, bem como o peso das folhas antes e depois de serem oferecidas aos insetos. Concluiu-se que houve preferência alimentar das lagartas em função da nutrição com que as plantas de E. grandis eram mantidas.
\end{abstract}

Palavras-chave: herbivoria, nutrição mineral, deficiência nutricional, deficiência mineral, macronutrientes.

\section{INFLUENCE OF NUTRITIONAL STATUS OF Eucalyptus grandis W. Hill ex Maiden ON DEFOLIATING CATERPILLAR Eupseudosoma involuta (LEPIDOPTERA - ARCTIIDAE) DIETARY PREFERENCE}

\begin{abstract}
This work aimed at investigating whether the consumption of eucalyptus seedling leaves by a herbivorous insect can be affected by the nutritional condition to which these plants have been submitted. To do so, leaves of Eucalyptus grandis W. Hill ex Maiden seedlings ( 9 months old) kept for 30 days in several nutrient solutions, were offered to Eupseudossoma involuta (Lepidoptera-Arctiidae) caterpillars. Nutrient solutions differed from each other by the presencelabsence of nitrogen, phosphorus and potassium. Control treatments consisted of a complete solution ( $N, P, K$ present) and a treatment consisting of distilled water. The following parameters were measured: size and number of $\boldsymbol{E}$. grandis leaves before and after having submitted to the treatments and leaf weight before and after they were offered to caterpillars. We concluded that the preference of caterpillars is a function of the nutritional conditions where the plants of $\boldsymbol{E}$. grandis were maintained.
\end{abstract}

Key words: herbivore, mineral nutrition, nutritional deficiency, mineral deficiency, macronutrients.

\footnotetext{
${ }^{1}$ Recebido para publicação em 01.7.2003 e aceito para publicação em 10.8.2004.

${ }^{2}$ Departamento de Biologia Vegetal, Universidade Federal de Viçosa. 36570-000, Viçosa, MG.
} 


\section{INTRODUÇÃO}

A fisiologia, a ecologia e a etologia dos insetos herbívoros, dentre outros aspectos de sua biologia, estão inseridos dentro de um contexto nutricional. A qualidade e a quantidade de alimento ingerido, bem como a proporção entre elementos químicos e a ingestão de compostos do metabolismo secundário das plantas, podem causar maiores ou menores efeitos, interferindo inclusive na capacidade reprodutiva desses indivíduos (HAGEN et al., 1984) e, por conseqüência, na dinâmica de suas populações (PRICE, 1997).

Os insetos herbívoros correspondem a cerca da metade do total de insetos existentes, e a maior parte deles se alimenta de vegetais superiores (PIZZAMIGLIO, 1991), estando sujeitos a todas as alterações que resultam das interações das plantas com o meio ambiente (OSBORNE, 1973). A despeito disso, têm alto potencial reprodutivo, causando prejuízos principalmente na agricultura e em sistemas florestais de monocultivo (BARBOSA e SCHULTZ, 1987).

Segundo Fenny (1976) e Rhoades e Cates (1976), as espécies lenhosas perenes investem mais energia na produção de compostos de defesa. Porém, existem evidências de que os mecanismos de defesa dessas plantas podem ser modificados por estresses, inclusive de natureza nutricional (CHEW e ROADMAN, 1979).

A teoria atual da dinâmica de populações de fitófagos preconiza que plantas estressadas produzem alimentos com baixos teores de compostos químicos de defesa (RHOADES, 1979), e sabe-se também que a seleção da planta hospedeira pelo inseto é controlada por fatores químicos de ambos (CHAPMAN, 1982; PROKOPY e OWENS, 1983; PIZZAMIGLIO, 1991).

Com vistas no que foi anteriormente exposto, o objetivo deste trabalho foi investigar a existência de relação entre a condição nutricional em plantas de Eucalyptus grandis e o consumo de suas partes por lagartas Eupseudossoma involuta (Lepidoptera Arctiidae).

\section{MATERIAL E MÉTODOS}

As mudas de $E$. grandis foram obtidas ainda em tubetes no viveiro do IEF/ UFV. Escolheram-se aquelas que aparentavam maior vigor: $32,5 \mathrm{~cm}$ de altura em média, com número de folhas superior a 6 e que não apresentavam enovelamento das raízes. No viveiro, o cuidado com essas plantas estava restrito à aspersão diária com água.

Foram construídos vasos de Leonard com duas garrafas "pet" com capacidade para 21 cortadas, uma das quais contendo o substrato preparado com areia lavada e vermiculita $(1: 1)$ e, a outra, adaptada como coletor do excedente da solução nutritiva. Cada vaso de Leonard recebeu uma muda de $E$. grandis, e as plantas assim preparadas foram submetidas a cinco tratamentos com cinco repetições cada, estabelecidos de acordo com a presença ou ausência dos elementos nitrogênio, fósforo e potássio em soluções nutritivas, formuladas segundo Hoagland e Arnon (1950), como expresso no Quadro 1.

Quadro 1 - Relação dos respectivos tratamentos estabelecidos e dos reagentes utilizados na preparação das soluções nutritivas, segundo Hoagland e Arnon (1950). Completa = solução nutritiva completa, Sem N = solução nutritiva sem nitrogênio, Sem $\mathrm{P}=$ solução nutritiva sem fósforo, Sem K = solução nutritiva sem potássio e Água = água destilada sem acréscimo de elementos minerais

Table 1 - List of treatments and the reagent used in the preparation of the nutrient solutions according to Hoagland e Arnon (1950). Completa = nutritive solution with all mineral nutrients; Sem $N=$ nutritive solution without nitrogen; $\operatorname{Sem} P=$ nutritive solution without phosphorus; Sem K= nutritive solution without potassium; Água = distilled water without mineral nutrient

\begin{tabular}{lccccc}
\hline & \multicolumn{5}{c}{ Soluções nutritiva } \\
\cline { 2 - 6 } Reagentes & Completa & Sem & Sem & Sem & Água* \\
& & $\mathrm{N}$ & $\mathrm{P}$ & $\mathrm{K}$ & \\
\hline $\mathrm{KH}_{2} \mathrm{PO}_{4} 1 \mathrm{M}$ & $\mathrm{X}$ & & & & \\
$\mathrm{KNO}_{3} 1 \mathrm{M}$ & $\mathrm{X}$ & & $\mathrm{X}$ & & \\
$\left.\mathrm{Ca} \mathrm{NO}_{3}\right)_{2} 4 \mathrm{H}_{2} \mathrm{O} 1 \mathrm{M}$ & $\mathrm{X}$ & & $\mathrm{X}$ & $\mathrm{X}$ & \\
$\mathrm{MgSO}_{4} 1 \mathrm{M}$ & $\mathrm{X}$ & $\mathrm{X}$ & $\mathrm{X}$ & $\mathrm{X}$ & \\
$\mathrm{K}_{2} \mathrm{SO}_{4} 0,5 \mathrm{M}$ & & $\mathrm{X}$ & & & \\
$\mathrm{Ca}\left(\mathrm{H}_{2} \mathrm{PO}_{4}\right) 0,05 \mathrm{M}$ & & $\mathrm{X}$ & & $\mathrm{X}$ & \\
$\mathrm{CaSO}_{4} 0,01 \mathrm{M}$ & & $\mathrm{X}$ & & & \\
\hline
\end{tabular}

*Foi utilizada água destilada como tratamento-testemunha. 
Com exceção da testemunha, todas as soluções nutritivas receberam acréscimo de tartarato de $\mathrm{Fe} 0,5 \%$ e micronutrientes na forma de $\mathrm{H}_{3} \mathrm{BO}_{3}, \mathrm{MnCl}_{2} \cdot 4 \mathrm{H}_{2} 0, \mathrm{ZnSO}_{4}$. $7 \mathrm{H}_{2} \mathrm{O}, \mathrm{CuSO}_{4} 5 \mathrm{H}_{2} 0, \mathrm{H}_{2} \mathrm{MoO}_{4} \mathrm{H}_{2} 0$, segundo Hoagland e Arnon (1950).

As plantas permaneceram sob os tratamentos por 30 dias. Inicialmente, cada vaso recebeu $250 \mathrm{ml}$ de solução nutritiva, segundo o tratamento estabelecido, e durante o período experimental foram acrescentados $100 \mathrm{ml}$ da respectiva solução em cada vaso, a cada dois dias, descartando-se o excedente contido nos recipientes coletores.

Avaliou-se a resposta das plantas aos tratamentos, através dos parâmetros altura e número de folhas.

Os indivíduos de Eupseudosoma involuta (Lepidoptera - Arctiidae) provieram do Insetário da Universidade Federal de Viçosa, onde sua dieta diária constava somente de folhas de eucalipto e estavam no último estágio de desenvolvimento quando foram utilizadas no experimento.

Ao final de 30 dias, as plantas de $E$. grandis foram medidas na sua altura e as folhas, contadas, colhidas e pesadas a fresco.

O material vegetal obtido de cada planta foi oferecido a somente uma lagarta e permaneceu à sua disposição por um período de 20 horas, em ambiente reservado, à temperatura ambiente de aproximadamente $20{ }^{\circ} \mathrm{C}$.

Para verificar a perda de peso das folhas devido à dessecação, foram obtidos discos foliares de $9 \mathrm{~mm}$ em cada tratamento, que foram pesados e mantidos dentro dos recipientes com as respectivas folhas intactas e as lagartas. Decorrido o tempo experimental, os discos foliares foram novamente pesados, assim como as folhas restantes.

O consumo de massa foliar pelas lagartas foi obtido da seguinte forma: do peso inicial das folhas foi subtraído o peso final. Desse resultado, foi subtraída a perda de peso devida à dessecação, obtida pelo resultado da diferença entre os pesos inicial e final dos discos foliares.

Os dados obtidos foram submetidos à análise de variância. Constatada a significância dos tratamentos, foi feita a comparação de médias pelo teste $t$ de
Student a $1 \%$ de significância estatística.

\section{RESULTADOS E DISCUSSÃO}

Foram observadas nas plantas de Eucalyptus grandis, segundo os respectivos tratamentos, alterações morfológicas indicando sintomas de deficiência dos macronutrientes $\mathrm{N}, \mathrm{P}$ e K.

As diferenças verificadas entre os dados dos tratamentos foram significativas, nos parâmetros altura e número de folhas. As plantas submetidas à solução nutritiva sem potássio apresentaram melhores resultados com relação a esses parâmetros (Quadro 2).

O maior desenvolvimento das mudas de $E$. grandis sob tratamento com solução nutritiva sem K também foi relatado por Brendolan, Pellegrini e Alves (2000). Esse efeito parece estar relacionado com a fisiologia do desenvolvimento inicial da espécie em questão, porém não foram encontrados outros trabalhos relatando tais resultados.

O consumo das folhas de E. grandis pelas lagartas de Eupseudossoma involuta indicou ser estatisticamente diferente entre os tratamentos, havendo maior herbivoria das folhas cujas plantas foram mantidas em solução nutritiva sem acréscimo de potássio $\left(\mathrm{F}_{5,19}=5,8364, \mathrm{P}<0,0028\right)$, em relação aos demais tratamentos (Figura 1).

Quadro 2 - Altura média das mudas de E. grandis $(\mathrm{cm})$, crescimento médio em relação á altura inicial (cm) e número médio de folhas por planta, no final do experimento. Comparação das médias pelo teste $\mathrm{t}$ de Student ( $\mathrm{alfa}=0,01)$. As médias na mesma coluna seguidas por pelo menos uma mesma letra não diferem estatisticamente entre si

Table 2 - Size average of the E. grandis seedlings ( $\mathrm{cm})$, growth average relative to initial size $(\mathrm{cm})$ and number of leaves average per plant at the end of the experiment. Means compared by $t$ Student test (alpha $=0,01)$. Means in the same column followed by the same letter do not differ statisticaly

\begin{tabular}{lccc}
\hline Tratamentos & Altura final & Crescimento & $\mathrm{N}^{\circ}$ de folhas \\
\hline 1 -Completa & $34.44 \mathrm{bc}$ & 1.94 & $37.4 \mathrm{ac}$ \\
2 -Sem N & $38.94 \mathrm{ac}$ & 6.44 & $23.8 \mathrm{bc}$ \\
3 -Sem P & $38.7 \mathrm{ac}$ & 6.20 & $42.8 \mathrm{ac}$ \\
4 -Sem K & $44.12 \mathrm{ac}$ & 11.62 & $51.6 \mathrm{a}$ \\
5 -Água & $36.62 \mathrm{bc}$ & 4.12 & $18.4 \mathrm{bc}$ \\
\hline
\end{tabular}

R. Árvore, Viçosa-MG, v.28, n.5, p.749-754, 2004 


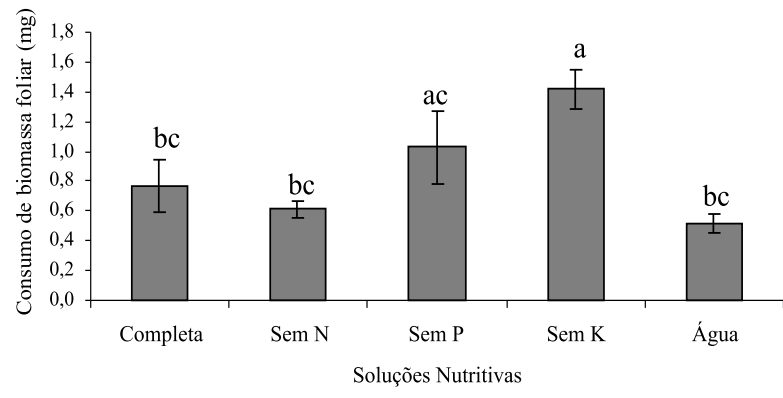

Figura 1 - Consumo médio (g) de folhas de plantas de Eucalyptus grandis, mantidas em soluções nutritivas com diferentes formulações, por lagartas de Eupseudossoma involuta (Lepidoptera - Arctiidae). Completa $=$ solução nutritiva completa, Sem N = solução nutritiva sem nitrogênio, Sem $\mathrm{P}$ = solução nutritiva sem fósforo, Sem K = solução nutritiva sem potássio e Água = água destilada sem acréscimo de elementos minerais. As barras seguidas de pelo menos uma mesma letra não diferem estatisticamente entre si, pelo teste t de Student (alfa $=0,01)$.

Figure 1 -Consumption ( $g$ ) of leaves of Eucalyptus grandis plants maintained in nutrient solutions with different formulations by caterpillars of Eupseudossoma involuta (Lepidoptera - Arctiidae). Completa $=$ nutritive solution with all mineral nutrients, Sem $N=$ nutrient solution without nitrogen, Sem $P=$ nutrient solution without phosphorus, Sem $K=$ nutrient solution without potassium and Water $=$ water distilled without increment of mineral elements. Bars followed by the same letter do not differ statisticaly by the of $t$ Student test (alpha $=0,01$ ).

Segundo a hipótese do balanço carbono/nutriente, proposta por Bryant et al. (1983) para explicar aspectos da interação entre as plantas e os herbívoros, a deficiência de nutrientes essenciais leva ao declínio da taxa fotossintética e à diminuição do crescimento e valor nutricional da planta como fonte de alimento para os herbívoros (PRICE, 1997). No entanto, Wheeler, et al. (1998), argumentaram que, em condições de deficiência nutricional, pode haver aumento do consumo de partes das plantas pelos herbívoros, como estratégia para compensar a baixa aquisição de nutrientes. Segundo Herms (2002), a variação na síntese de compostos de defesa em relação à disponibilidade de nutrientes descreve uma curva parabólica, em que ambos os extremos da condição nutricional correspondem à menor atividade do metabolismo secundário.

R. Árvore, Viçosa-MG, v.28, n.5, p.749-754, 2004
Nas plantas deficientes em K ocorre menor síntese de substâncias químicas de defesas, diminuindo principalmente o teor de compostos fenólicos. Fisicamente, esses indivíduos têm as paredes celulares e a cutícula foliar menos engrossadas e rijas (PERRENOUD, 1990). O acúmulo de compostos de baixo peso molecular altera o equilíbrio osmótico celular e favorece o acúmulo de putrescina, o aumento do teor de nitrogênio alfa-amínico e de ácidos orgânicos nos tecidos (MALAVOLTA, 1989, KOCHIAN, 2000, SILVEIRA e MALAVOLTA, 2000). Portanto, as plantas com deficiência nutricional de potássio são nutricionalmente mais interessantes e apresentam menos defesas. Entrentanto, N e P são elementos de extrema importância nutricional para as plantas e para os animais. A ausência de $\mathrm{N}$ acarreta a liberação de açúcares e o aumento da pressão osmótica celular, enquanto a falta de $\mathrm{P}$ aumenta a relação $\mathrm{P}_{\text {orgânico }} / \mathrm{P}_{\text {inorgânico }}$ (MALAVOLTA, 1989; KOCHIAN, 2000) e diminui a síntese protéica. A grande exigência metabólica de proteínas pelas lagartas, que se encontravam em um período de crescimento (WIGGLESWORTH, 1972; GULLAN e CRANSTON, 1994), pode ter sido o fator decisivo na preferência alimentar desses animais, que, em consequiência, consumiram menos biomassa das plantas de eucalipto não supridas com $\mathrm{N}$ e $\mathrm{P}$.

Kaul, et al. (1968) relataram que, em um experimento para o diagnóstico de deficiência nutricional em mudas de $E$. grandis conduzido nas mesmas condições deste, também houve maior ataque de insetos às plantas mantidas sob nutrição deficiente em K. Dessa forma, os resultados indicaram que o maior consumo das folhas de E. grandis submetidas à carência de K pelas lagartas de E. involuta se deveu a dois fatores principais: alto valor nutricional da dieta e diminuição de defesas físicas e químicas. As plantas nessa condição foram supridas com nitrogênio e fósforo, acarretando alta concentração de nitrogênio e fósforo livres, $\mathrm{e}$, concomitantemente, apresentavam tecidos mais moles e menor concentração de compostos químicos de defesa, devido à ausência de $\mathrm{K}$.

Concluiu-se que a condição nutricional das mudas de Eucalyptus grandis interferiu na preferência alimentar das lagartas de Eupseudossoma involuta, sendo plantas deficientes em $\mathrm{K}$ mais suscetíveis à herbivoria. 


\section{REFERÊNCIAS BIBLIOGRÁFICAS}

BARBOSA, P.; SCHULTZ, J. C. Insect outbreaks. San Diego: Academic Press, 1987. p.217-233.

BRENDOLAN, R.A.; PELLEGRINI, M.T.; ALVES, P.L.C.A. Efeitos da nutrição mineral na competição inter e intraespecífica de Eucalyptus grandis e Brachiaria decumbens: 1- crescimento. Scientia Forestalis, n.58, p.49-57, 2000.

CHAPMAN, R.F. The insects: structure and function. Cambridge: Harvard University Press, 1982.919p.

CHEW, F.S.; ROADMAN, J.E. Plant resources for chemical defense. In: ROSENTHAL, G.A.; JANZEN, D. (Ed.) Herbivores: their Interactions With Secondary Plant Metabolites. New York: Academic Press, 1979. p.271-307.

FENNY, P. Plant apparency and chemical defense. In: WALLACE, J.W.; MANSELL, R.L. (Eds.) Biochemical interactions between plants and insects. Recent Advances in Phytochemistry. New York: Plenum Press, 1976. p.1-40.

GOMES, J.M. et al. Crescimento de mudas de Eucalyptus grandis em diferentes tamanhos de tubetes e fertilização N-P-K. Revista Árvore, v.27, n.2, p.113-27, 2003.

GULLAN, P.J.; CRANSTON, P.J. The insects: An outline of entomology. London: Chapman \& Hall, 1994. 491p.

HAGEN, K. S. et al. The food of insects. In: HUFFAKER, C.B.; RABB, R.L. (Eds.) Ecological Entomology. New York: John Willey \& Sons, 1984. p.79-112.

HERMS, D.A. Effects of fertilization on insect resistance of woody ornamental plants: reassessing an entrenched paradigm. Environmental Entomology, v.31, n.6, p.923-933, 2002.
HOAGLAND, D.R.; ARNON, D.I. The waterculture method for growing plans without soil. Berkley: The college of Agriculture/University Of California, 1952. p.32

KAUL, O.N., SRIVASTAVA, P.B.L.; TANDON, V.N. Nutrition studies on Eucalyptus. III. Diagnosis of mineral deficiencies in Eucalyptus grandis seedlings. Indian Forester, v.94, n.11, p.831$834,1968$.

KOCHIAN, L.V. Molecular physiology of mineral nutrient acquisition, transport and utilization. In: BUCHANAN, B.B.; GRUISSEM, W .; JONES, R.L. (Eds.) Biochemistry and Molecular Biology of plants. Maryland: American Society of Plant Physiologists, 2000. 1204-1247p.

MAlavolta, E. et al. Avaliação do estado nutricional das plantas. Princípios e aplicações. Piracicaba: POTAFÓS, 1989. p.201.

OSBORNE, D.J. Mutual regulation of growth and development in plants insects. In: Van EMDEN, H.F. (Ed). Insect/plant relationships. London: Oxford Blackwell, 1973. p.33-42.

PERRENOUd, S. Potassium and plant health. 2.ed. Berna: International Potash Institute, 1990. 363p.

PIZZAMIGLIO, M.A. Ecologia das Interações Inseto/Planta. In: PANIZZI e PARRA (Eds.). Ecologia nutricional de insetos $e$ manejo integrado de pragas. Brasilia: Manole/CNPq, 1991.p.101-129.

PRICE, P.W. Insect ecology. New York: John Wiley \& Sons, 1997.p. 73-138.

PROKOPY, R.J.; OWENS, E.D. Visual detection of plants by herbivorous insects. Annual Review Entomology, v.28, p.337-64, 1983.

RHOADES, D.F.; CATES, R.G. Towards a general theory of plant antiherbivore chemistry. In: WALLACE, J.W.; MANSELL, R.L. (Eds.) Biochemical interactions between plants and insects. Recent Advances in Phytochemistry, New York: Plenum Press, 1976. p.168-213.

R. Árvore, Viçosa-MG, v.28, n.5, p.749-754, 2004 
RHOADES, D.F. Evolution of chemical defenses against herbivores: In: ROSENTHAL, G.A.; JANZEN, D. (Eds.) Herbivores: their interactions with secondary plant metabolites. New York: Academic Press, 1979. p.3-54

SILVEIRA, R.L.V.; MALAVOLTA, E. Nutrição e adubação potássica em Eucalyptus. Informações Agronômicas, n.91, p.1-12, 2000. (Encarte TécnicoPotafós).
WHEELER, G.S.;VAN, T.K.; CENTER, T.D. Herbivore adaptations to low-nutrtiente food: weed biological control specialist Spodoptera pectinicornis (Lepidoptera - Noctuidae) fed the floating aquatic plant Pistia stratiotes.

Environmental Entomology, v.27, n.3, p.997-1000,1998.

WIGGLESWORTH, V.B. The principles of insect physiology. New York: Chapman \& Hall, 1972. 269p. 